\title{
Bufalin Inhibits the Differentiation and Proliferation of Cancer Stem Cells Derived from Primary Osteosarcoma Cells through Mir-148a
}

\author{
Yuewen Chang Yongfang Zhao Wei Gu Yuelong Cao Shuqiang Wang \\ Jian Pang Yinyu Shi
}

Department of Orthopedics and Traumatology, Shuguang Hospital affiliated to Shanghai University of Traditional Chinese Medicine, Shanghai, China

\section{Key Words}

Cancer Stem Cells • Osteosarcoma • MicroRNAs • Bufalin

\begin{abstract}
Background/Aims: Osteosarcoma (OS) is the second leading cause of cancer-related death in children and young adults. Chemoresistance is the most important cause of treatment failure in OS, largely resulting from presence of cancer stem cells (CSCs). However, CSCs isolated from cancer cell lines do not necessarily represent those from primary human tumors due to accumulation of genetic aberrations that increase with passage number. Therefore, studies on CSCs from primary OS may be more important for understanding the mechanisms driving the chemoresistance of CSCs in OS. Methods: We established a primary culture of OS cells, known as C1OS, from freshly resected tumor tissue. We further isolated CSCs from C1OS cells (C1OSCSCs). We analyzed the effects of bufalin, a traditional Chinese medicine, on the stemness of C1OS-CSCs. We also analyzed the microRNA (miR) targets of bufalin on the stemness of C1OSCSCs. Moreover, we examined these findings in the OS specimen. Results: Bufalin inhibited the stemness of C1OS-CSCs. Moreover, we found that miR-148a appeared to be a target of bufalin, and miR-148a further regulated DNMT1 and p27 to control the stemness of OS cells. This mechanism was further confirmed in OS specimen. Conclusion: Our data suggest that bufalin may be a promising treatment for OS, and its function may be conducted through regulation of miR-148a.
\end{abstract}




\section{Cellular Physiology Cell Physiol Biochem 2015;36:1186-1196 \begin{tabular}{l|l} 
and Biochemistry Published online: June 25, 2015 & $\begin{array}{l}\text { C) 2015 S. Karger AG, Basel } \\
\text { www.karger.com/cpb }\end{array}$ \\
\hline
\end{tabular} \\ Chang et al.: Bufalin Inhibits Cars from Os Cells Through Mir-148a}

\section{Introduction}

Osteosarcoma (OS) is a type of solid bone cancer mainly arising in young adults. It account for approximately $2.4 \%$ of all malignancies in young adults and is the second leading cause of cancer-related death of pediatric patients [1-3]. The standard care for OS today is based on a combination of aggressive surgical resection and different pre- and post-operative drugs, but it can provide just 65-70\% 5-year survival rate without metastasis for OS patients [4-10]. Therefore, a great need for new treatments for osteosarcoma exits. However, the underlying cellular mechanisms of the development of OS chemoresistance and metastasis remain unclear.

Recently, it has been suggested that within a heterogeneous tumor there was a small subpopulation of cells called cancer stem cells (CSCs) that are responsible for chemoresistance and tumor metastasis [11]. Tumor shrinkage can be achieved by traditional treatment patterns, but most tumors will recur after treatment because of CSCs which could survive and then regenerate tumor growth $[12,13]$. Specifically targeting the CSC population may be a more effective therapy strategy. Since the proposal of the CSC hypothesis, a lot of studies have been performed to isolate and identify OS stem cells using various methods [14-17]. In a recent study, we derived CSCs from the human hMG63 osteosarcoma cell line using a new strategy combining a suspension culture system with the chemotherapy drug cisplatin [18]. We also detected the expression of classic stem cell markers and confirm stem cell-like properties of hMG63 derived cancer stem cells.

Bufalin is the major component of the Chinese medicine Chan $\mathrm{Su}$. It is an extract of dried toad venom from the skin glands of Bufo gargarizans. Previous studies suggest that bufalin inhibits the proliferation of transplanted human hepatocellular carcinoma in nude mice [19]. In addition, it has been reported that bufalin induces apoptosis in various human cancer cell lines, including leukemia [20], prostate cancer [21], gastric cancer [22], and osteosarcoma [23]. We also reported its potent impact on human osteosarcoma CSCs.

MicroRNAs, a type of noncoding small RNAs of 19-25 nt, can repress translation and induce cleavage of mRNA by binding to the 3'-untranslated region (UTR) of the target genes. MicroRNAs play important roles in multiple physiological and pathological functions, including development, differentiation, cell proliferation, apoptosis, and stress responses $[24,25]$. In addition, recent evidence suggests that some microRNAs are involved in OS tumorigenesis and chemoresistance. For example, the inhibition of miR-34c significantly stimulated OS cell invasion and chemoresistance in vitro. In contrast, restoring miR-34c inhibited OS cell invasion and chemoresistance [26]. miR-33a promotes OS cell resistance to cisplatin by down-regulating TWIST. Inhibition of miR-33a by antagomir-33a enhances cisplatin-induced apoptosis in OS cells by up-regulating TWIST expression [27]. The expression of miR-21 regulates cellular processes in osteosarcoma, perhaps through regulating RECK [28]. Down-regulation of miR-143 was found in both osteosarcoma cell lines and primary tumor samples. The restoration of miR-143 could reduce cell viability and promote cell apoptosis through repression of Bcl-2 [29]. miR-140 was found to play a role in chemosensitivity to chemicals such as 5-fluorouracil (5-FU) in osteosarcoma. Sensitivity to 5-FU treatment can be obtained by blocking expression of miR-140 in cancer cells; on the other hand, the overexpression of miR-140 made tumor cells more resistant. It suggested miR-140 as a potent target to overcome drug resistance [30]. Given the numerous studies, the mechanism details underlying the interaction of OS CSCs and miRNA are still not well understood.

In this study, we successfully established a primary culture of OS cells, named C1OS, from freshly resected tumor tissue. Then, we derived cancer stem cells from C10S cells using methods we described previously [18]. Similar to the cancer stem cells that we derived from the hMG63 cell line in a previous study, the cancer stem cells from C1OS cells can respond to bufalin. We also found a significantly upregulated expression of miR-148a in bufalin-treated primary cancer stem cells derived from C10S. Ectopic overexpression of miR148a inhibits the stemness of cancer stem cells by suppressing expression of DNMT1 directly. 


\section{Methods and Materials}

\section{Establishment of primary osteosarcoma cell lines}

This study was approved by ethical committee of Shanghai University of Traditional Chinese Medicine. After receiving signed informed consent forms, human cancer tissues were obtained from patients with primary osteosarcoma. Tumor tissue obtained from patients was washed in medium (DMEM supplemented with penicillin-streptomycin) and then minced into small pieces by scissors. The tissues were then resuspended in culture medium (DMEM medium supplemented with 10\% FBS) and explanted to dishes pre-coated with collagen. Explants with epithelial monolayer outgrowth were preserved and transferred to a new dish for subculture. Serial subculture was performed to obtain primary tumor cell lines. All primary tumor cells were maintained at $37^{\circ} \mathrm{C}$ in a humidified incubator with $5 \%$ $\mathrm{CO}_{2}$.

\section{Animals}

This study was approved by the institutional animal care and use committee of Shanghai University of Traditional Chinese Medicine. Nude mice were purchased from Shanghai Laboratory Animal Company. Mice were housed under pathogen-free conditions according to the standard guidelines of the institutional animal care and use committee of Shanghai University of Traditional Chinese Medicine. For xenografts formation assay, the C1OS colonies consisting of different number of cells were injected into nude mice. The tumor size was monitored.

Cell culture

hMG-63 cells were obtained from American type culture collection (ATCC) and stored in our laboratory. hMG-63 and C1OS were cultured in medium (DMEM supplemented with $10 \% \mathrm{FBS}$ ) at $37^{\circ} \mathrm{C}$ in a humidified incubator with $5 \% \mathrm{CO}_{2}$. The medium was changed every three days.

Plasmids construction and lentiviral infections

pLemiR-148a was obtained by cloning human pre-miR-148a sequence (amplified from genomic DNA) into pLemiR-tRFP vector. For the production of lentiviruses, specific vectors was transfected into $293 \mathrm{~T}$ cells by calcium phosphate. $48 \mathrm{hrs}$ after the transfection, supernatants were harvested, filtered with $0.45 \mu \mathrm{m}$ filters. These supernatants were used to infect cells in presence of Polybrene (Sigma-Aldrich).

\section{Realtime PCR}

Total RNA was isolated using the TRIzol Reagent (Invitrogen) according to manufacturer's protocol. Realtime PCR was performed on the LightCycle480 system (Roche) using SYBR Green Supermix (Takara). The realtime PCR reaction condition was 45 cycles of $30 \mathrm{~s}$ at $94^{\circ} \mathrm{C}$, $5 \mathrm{~s}$ at $94^{\circ} \mathrm{C}$ and $30 \mathrm{~s}$ at $60^{\circ} \mathrm{C}$.

\section{Immunohistochemistry}

Formalin-fixed samples were washed twice with PBS, followed by incubation with the blocking solution $(0.2 \%$ Triton- 100 and $5 \%$ goat serum in PBS) for $1 \mathrm{~h}$. Samples were then incubated with primary antibody and secondary antibody for $1 \mathrm{~h}$.

\section{Western blotting assay}

Whole cell lysates were prepared using pre-chilled RIPA. The samples were then centrifuged at $12000 \mathrm{~g}$ for $20 \mathrm{~min}$ at $4^{\circ} \mathrm{C}$ and supernatants were collected for protein concentration determination. The total proteins was separated on SDS-PAGE gel, and transferred onto a nitrocellulose membrane. The membrane was then incubated with the blocking solution, followed by incubation overnight with appropriate primary antibodies at 
$4^{\circ} \mathrm{C}$ and subsequently with secondary antibodies for $1 \mathrm{~h}$ at RT. The signals were visualized using LI-COR infrared imaging system (LI-COR) according to the manufacturer's guidelines.

\section{Flow Cytometry}

Dissociated cells were incubated with primary antibodies for $20 \mathrm{~min}$ on ice, and washed twice with PBS. Cells were then incubated with corresponding secondary antibodies for 20 min. After washing, flow cytometry was carried out using a FACSA flow cytometer (BD Immunocytometry Systems).The antibodies used were anti- CD133, anti -CD44, anti- CD90, each at a dilution of 1:40.

\section{Statistical Analysis}

All data quantification and statistical analysis were performed with SPSS 13.0 software. Data are presented as the mean \pm SEM.

\section{Results}

\section{Establishment of primary osteosarcoma cell line C1OS}

Freshly resected osteosarcoma samples were minced with scissors into small pieces and explanted to pre-coated dishes. One week later, explants with epithelial monolayer outgrowth formed a halo (Fig. 1A). These explants were transferred into a new dish for subculture. After serial cultures, we derived a primary tumor cell line termed C1OS (Fig. 1B). To test whether C1OS cells may well represent osteosarcoma, an array-based gene

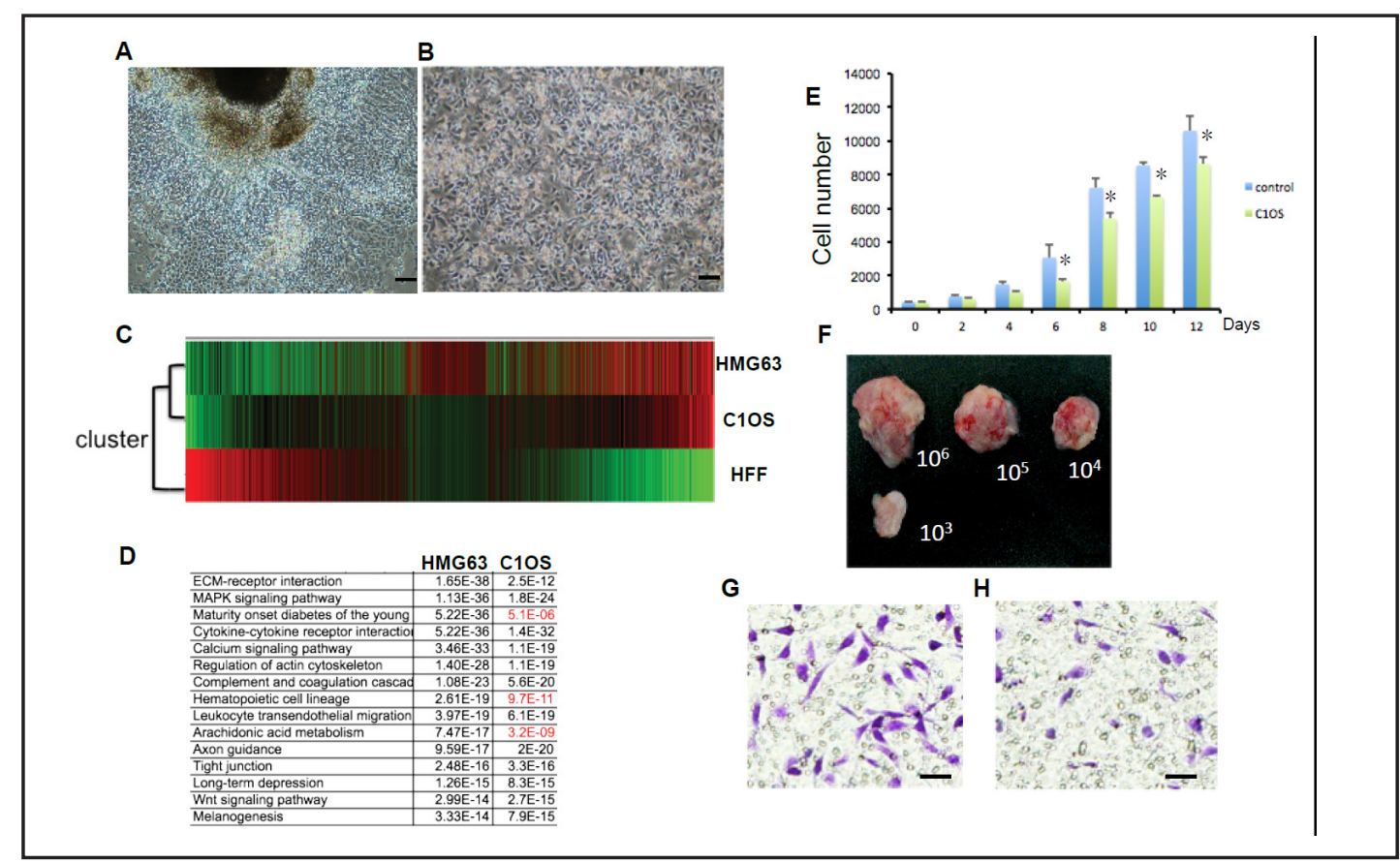

Fig. 1. Isolation and identification of the C1OS primary osteosarcoma cell line. (A) Osteosarcoma sample explant in culture for 1 week. Photomicrograph magnification, $\times 200$. (B) The C10S cell line in culture for 4 weeks at the second passage. Photomicrograph magnification, $\times 200$. (C) Self-organizing heat map of differentially expressed genes among the three cell lines. Induced genes are represented in red. Suppressed genes are represented in green. (D) Pathway analysis. For the 20 pathways that are most active in hMG63, there were 17 overlapping pathways in C1OS. The other 3 pathways also have significant differences compared with hMG63 (red). (E) Cell-glo assay of hMG63 (control) and C10S in culture. (F) The xenograft tumors formed by different amounts of C10S cells for 4 weeks. Scale bar is $1 \mathrm{~cm}$. (G) Transwell migration assays of C10S cells. (H) Transwell invasion assays of C10S cells. * $\mathrm{p}<0.05$.

\section{KARGER}




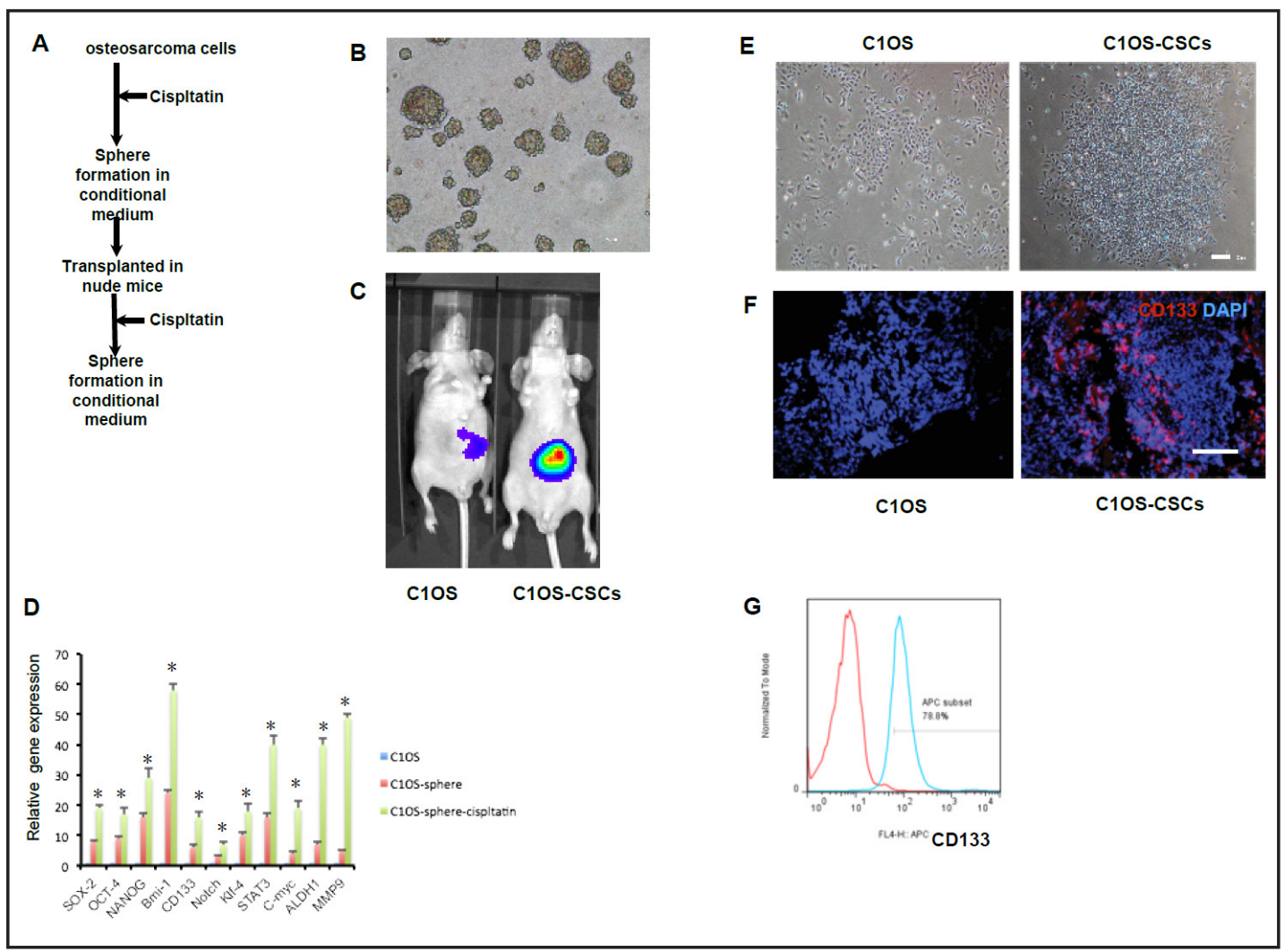

Fig. 2. Isolation of cancer stem cells from primary C1OS cells. (A) Workflow chart for isolating the cancer stem cells. (B) C10S formed spheres when grown in a suspension culture system for 14 days. (C) Nude mice bearing C1OS or C10S-CSCs tumor xenografts were imaged $24 \mathrm{~h}$ post-injection of the imaging probe. (D) The expression levels of the cancer stem cell markers in C1OS and C1OS-CSCs were determined by real time PCR. The data represent the mean \pm SEM. (E) C1OS and C10S-CSCs were seeded at equal confluence and allowed to grow for up to 18 days. We found tumor sphere-like colony formation in C10S-CSCs cells but not in C10S cells. (F-G) The expression levels of the surface marker CD133 were determined by immunohistochemistry (F) and flow cytometry (G). Scar bars are $50 \mu \mathrm{m}$.

expression profile analysis was performed. We found that the gene expression profile of C1OS was similar to the commercial human OS cell line MG63 but not to skin fibroblast cells (Fig. 1C). In addition, the altered signaling pathways of C1OS resembled MG63, as indicated by an analysis on the array data (Fig. 1D). Moreover, the proliferation ability of C1OS resembled MG63 (Fig. 1E).The malignant potential of the C1OS was evaluated by injection of different amounts $\left(10^{6}, 10^{5}, 10^{4}, 10^{3}\right.$, respectively) of C1OS cells into NOD/SCID mice. All four doses could form xenografts within 4 weeks after the injection, and the volume of the xenografts was correlated to the injection dose (Fig. 1F). The C1OS cell lines also displayed a significant ability to migrate and invade when tested using a trans-well system in vitro (Fig. 1G-1H). These data suggest that C1OS may well represent the original tumor.

\section{Cancer stem cells derived from C1OS cells}

Cancer stem cells were derived from C10S following a workflow (Fig. 2A). Briefly, we generated a tumor sphere in a suspension culture, which was then injected into nude mice. The CSC populations were enriched under selection stress from cisplatin, and these drugresistant cells were cultured in vitro to form cell colonies (Fig. 2A). The morphology of the acquired cancer stem cells could be seen in culture (Fig. 2B).

When injected into NOD/SCID mice at same amount $\left(10^{4}\right)$ as C1OS, C1OS-CSC formed a much bigger xenograft than C10S, suggesting that C1OS-CSC cells grow faster (Fig. 2C). We thus analyzed the expression patterns of several cell surface markers and confirmed the stem 


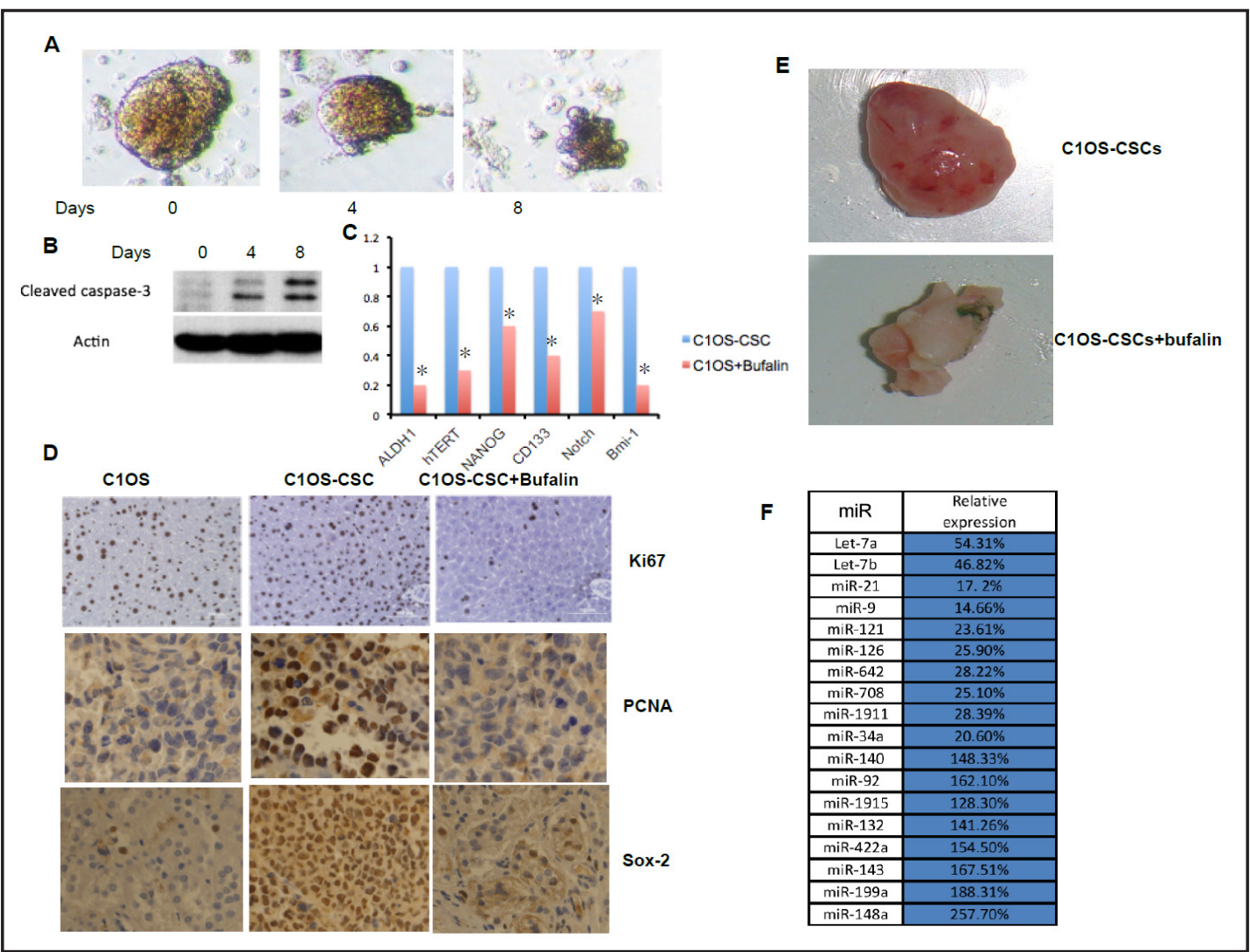

Fig. 3. Bufalin inhibits the stemness of C1OS-CSCs. (A) CSCs were plated on the CSC culture system, and the cells treated with bufalin before and after 4- and 8-day incubations was shown. (B) Cleaved caspase3 was determined by western blotting. (C) Transcript levels of cancer stem cell markers were measured in the C10S-CSCs cultured with bufalin or DMSO by quantitative PCR. (D) PCNA, Ki67 and sox-2 were measured in the C1OS and C1OS-CSCs cultured with or without bufalin by immunohistochemistry. (E) The xenograft tumor formation of C1OS-CSCs cultured with (up) or without bufalin (down). (F) miRNA profiling of C10SCSCs cultured with or without bufalin was performed using Affymetrix microarray analysis with miRNA array chips. Eight miRNAs were upregulated and ten were down-regulated in the C1OS-CSCs cultured with bufalin. ${ }^{*} \mathrm{p}<0.05$.

cell-like properties of the isolated C1OS-CSCs (Fig. 2D). Sphere formation and serial passages are hallmarks of CSCs. We found that C1OS-CSC was capable of forming spheres while C1OS cells failed to form spheres (Fig. 2E). CD133 was a surface marker of OS-CSCs [31]. We found that C10S-csc highly expressed CD133, by immunohistochemistry (Fig. 2F) and by flow cytometry (Fig. 2G). All these data suggest that the C1OS-CSC cells are cancer stem cells derived from primary tumor cells.

\section{Bufalin inhibits the proliferation of C1OS-CSCS}

Recently, we reported that bufalin can inhibit the differentiation, proliferation and sphere formation of hMG63 cancer stem cells [18]. To test whether bufalin affects C1OS-CSCs, $10 \mu \mathrm{M}$ bufalin was added to the culture of C10S-CSCs cells. We found that bufalin induced shrinkage of C1OS-CSC spheres over 8 days of culture (Fig. 3A). This shrinkage of CSC spheres may be a result of the activation of caspase-3, which suggested enhanced apoptosis (Fig. 3B). To further determine of the effects of bufalin on the stemness of C1OS-CSCs, we measured the levels of several classic stem cell markers, such as ALDH1, TERT, NANOG, CD133, Notch and Bim1, using quantitative real time PCR. In comparison to C1OS cultured with or without bufalin, the levels of stem cell markers were all down-regulated by the addition of bufalin (Fig. 3C). In addition, culturing with $10 \mu \mathrm{M}$ bufalin for 4 days was enough to significantly 
A
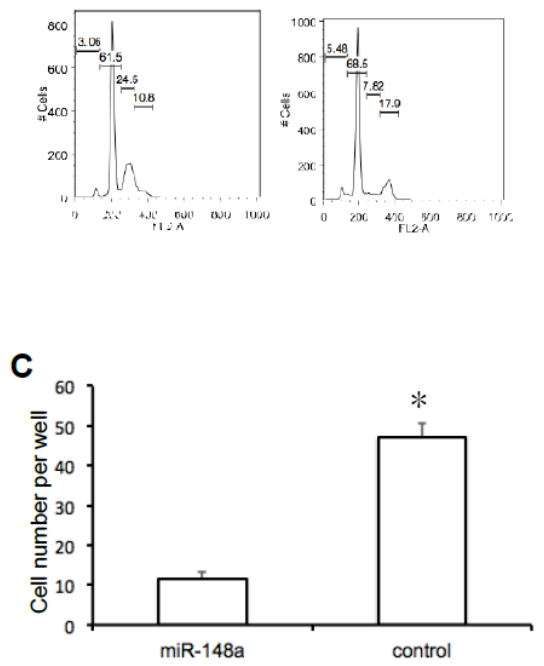

E

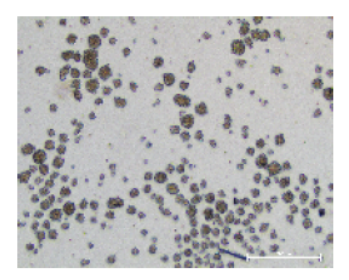

$\mathbf{F}$
B
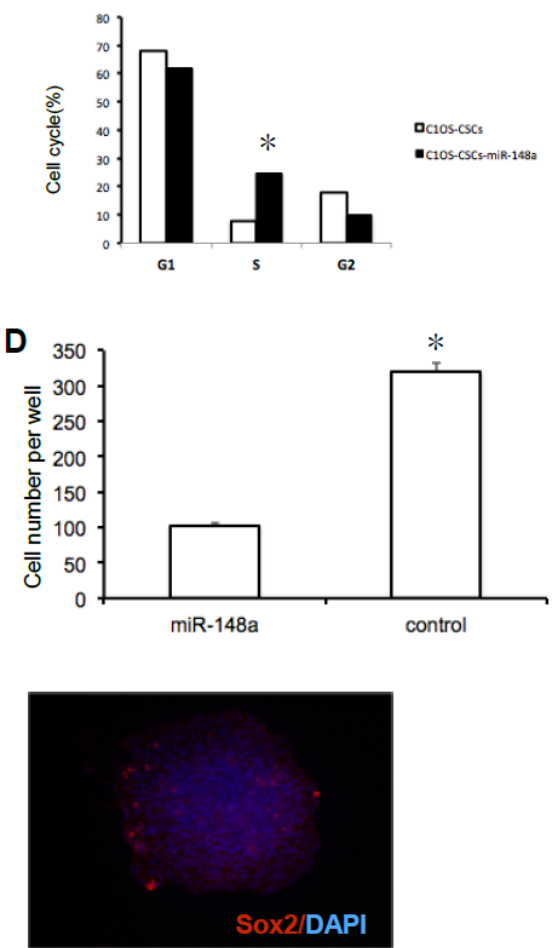

Fig. 4. Overexpression of miR148a inhibits the stemness of C10S-CSCs. (A-B) The effect of miR-148a on the cell cycle of C1OS-CSCs, shown by representative charts (A) and by quantification (B). (C) Quantitative analysis of transwell migration assays of C1OS cells transfected/untransfected with miR-148a. (D) Quantitative analysis of transwell invasion assays of C1OS cells transfected/untransfected with miR148a. (E) The sphere-like colony formation in C1OS-CSC cells was inhibited by transfection with miR-148a. (F) Expression of the stem cell marker SOX-2 was inhibited by transfection with miR-148a. (G) Sequence alignments of miR148a and its target sites in the 3'-UTR of DNMT1. (H) Expression of DNMT1 was inhibited by transfection with miR-148a. (I) Treatment with bufalin reduced the expression of DNMT1 in C1OS-CSCs. ${ }^{*} \mathrm{p}<0.05$.

down-regulate the levels of the proliferation marker ki67 and PCNA and the stem cell marker SOX-2 (Fig. 3D). When injected into NOD/SCID mice, a lower ability of xenograft formation was found in bufalin-treated C10S-CSCs compared to untreated C1OS-CSCs (Fig. 4E).

miRNAs were reported to be involved in carcinogenesis and stemness of OS cancer stem cell lines [32]. To identify the differentially expressed miRNAs, miRNA expression levels in C1OS-CSC cultured with or without bufalin expression was measured using a commercial microarray from Agilent (http://www.microrna.sanger.ac.uk/). miR-148a was found to have the greatest magnitude of increase in the 723 miRNAs tested after treatment with bufalin (Fig. 4F).

Overexpression of miR-148a significantly inhibits the stemness of C1OS-CSCS

Based on the above data, we postulated that overexpression of miR-148a may mimic the effects of bufalin on C10S-CSCs. To test this hypothesis, we expressed miR-148a in C10SCSCs using the pLemiR vector. We found that overexpression of miR-148a inhibited the G2/M transition in C1OS-CSCs, which suggests an impaired self-renewing ability (Fig. 4A-B). In addition, miR-148a overexpression also inhibited the migration and invasion abilities of C10S-CSCs (Fig. 4C-D).

Similar to bufalin, which can inhibit sphere formation in hMG63 cancer stem cells and C1OS-CSCs cultured in a non-adhesive culture system, overexpression of miR148a also inhibited the sphere formation of C1OS-CSCs (Fig. 4E). Moreover, the level of the stem cell 


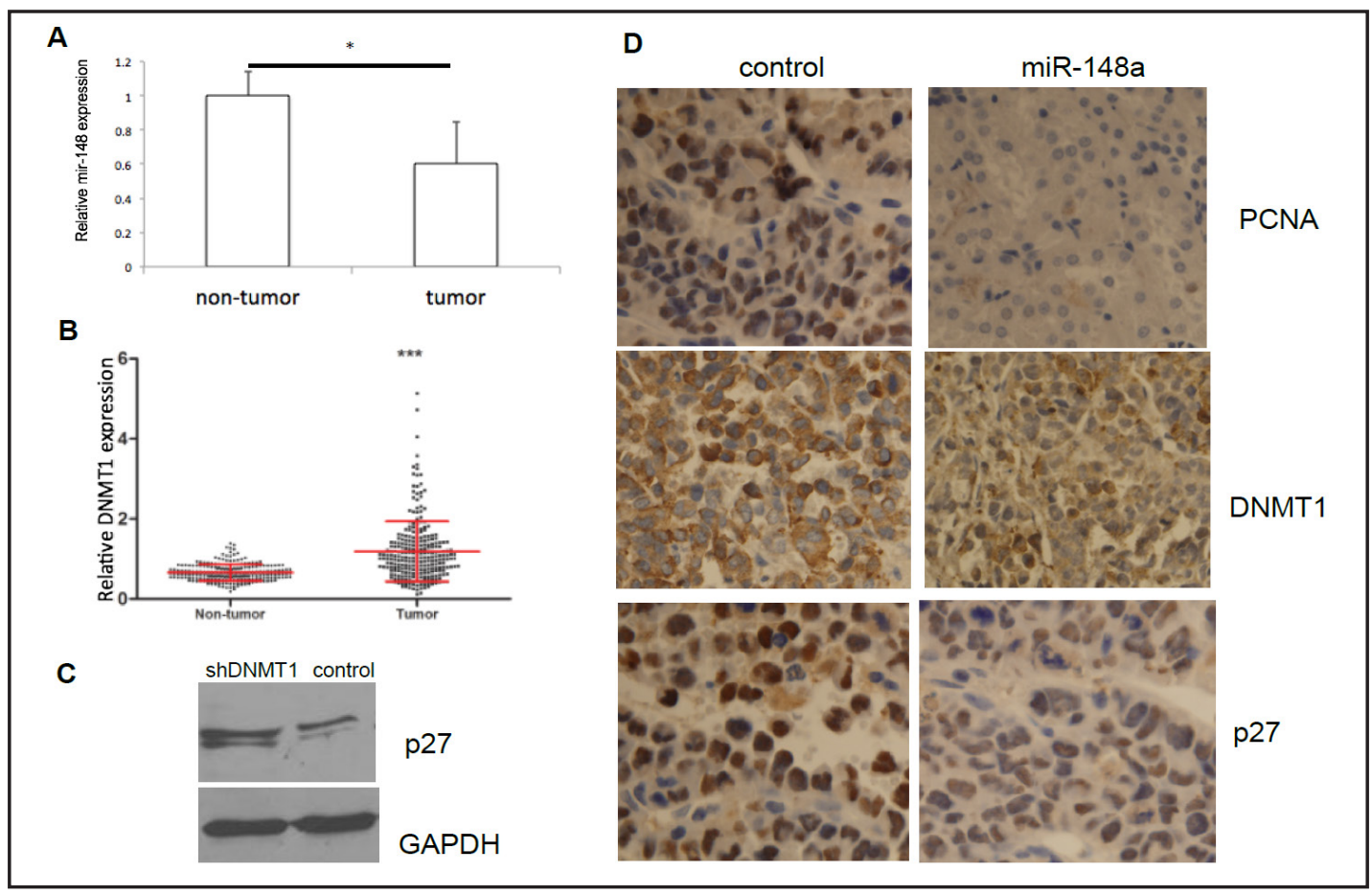

Fig. 5. DNMT1 is the target of miR-148a. (A) Expression levels of miR-148a in osteosarcoma tumor or non-tumor tissue measured by quantitative PCR. (B) The level of DNMT1 in osteosarcoma tumor or non-tumor tissue measured by quantitative PCR. (C) Expression level of p27 in osteosarcoma tumor or non-tumor tissue measured by western blotting. (D) Representative immunohistochemistry images for miR-148a of tumors formed in NOD-SCID mice or untreated C1OS-CSCs. ${ }^{*} \mathrm{p}<0.05$.

marker Sox-2 was also down-regulated by ectopically expression of miR-148a (Fig. 4F). To investigate the mechanism of miR-148a on C1OS-CSCs, we used a miRNA-target prediction program and found that a putative target gene of miR-148a was DNMT1 (Fig. 4G). We detected a repression of miR-148a on the expression of DNMT1 in C10S-CSCs expressing miR-148a (Fig. 4H). Interestingly, treatment of C1OS-CSCs with bufalin also repressed the expression of DNMT1 (Fig. 4I). Taken together, these data suggest that bufalin targets C1OSCSCs by repressing the expression of DNMT1 through inducing the expression of miR-148a.

\section{The association of miR148a with human osteosarcoma}

We have identified a critical role for miR-148a in cancer stem cells derived from primary OS cells. Then we further explored the association of miR-148a with human osteosarcoma. We found that the expression level of miR-148a in human osteosarcoma samples was approximately $60 \%$ of non-tumor tissues (Fig. 5A). DNMT1, the target of miR148a, was upregulated in these human samples (Fig. 5B). To identify the downstream target of DNMT1, we knocked down the expression of DNMT1 in C1OS-CSCs cell lines by siRNA. The expression levels of p27, a cyclin-dependent kinase inhibitor, were higher following the down-regulation of DNMT1 (Fig. 5C). In addition, inverse expression levels of DNMT1 and p27 were detected in the tumor formed in NOD-SCID mice by C1OS-CSCs treated with or without miR-148a (Fig. 5D).

\section{Discussion}

Over the past several decades, the survival rates of osteosarcoma patients have failed to improve and have reached a plateau, despite accumulating studies that have greatly increased our knowledge of this aggressive malignancy [33]. This is partially because the cellular mechanisms that lead to the development of OS chemoresistance and metastasis KARGER 
remain unclear. The emerging CSC theory has shed light on the study of the pathobiology of this disease. Based on this understanding, the strategy with which osteosarcoma is treated will also be updated. A new strategy that combines the CSC-targeted therapy and traditional chemotherapy is needed.

The base of developing a CSC-targeted therapy is the isolation of the tumor-type specific CSCs. Various methods, such as tumor spheres, sorting cells according to specific surface markers, side population cell sorting and other methods, were reported to be used in isolation and identification of osteosarcoma stem cells [14-17]. However, all these studies were based on established commercial cancer cell lines, whose ability to represent primary human tumors is limited by the genetic alternations that occur during increasing cultured period $[34,35]$. We also need patient-derived primary tumor cells for the development of personalized therapy [36-38]. In this study, we successfully established a primary culture of OS cells, termed C10S, from freshly resected tumor tissues, and derived a line of cancer stem cells from these primary cells. Because of their similar nature to tumor tissue, the establishment of cancer stem cells from primary tumors will facilitate the screening of new drugs specific to CSCs. However, taking the variability of patients into consideration, it is difficult to cover all the possible conditions by single cancer cell lines. More patient-derived primary tumor cell lines are needed in future studies.

A question arose once we have osteosarcoma CSC in hand. How can these CSCs be targeted and killed? miRNAs may play important roles in development of cancer [39]. However, targeting miRNAs have limitations on the treatment of human cancer because of the lack of appropriate in vivo delivery systems. To overcome such limitations, agents that can regulation expression levels of mRNA was used to target the CSCs. Natural agents could be a safer choice to be combined with conventional chemotherapeutics because of their nontoxic nature.

Our group has reported that bufalin can inhibit the proliferation and stemness of cancer stem cells derived from primary OS cells or the hMG63 cell line [18]. We compared the expression profile of miRNA in cancer stem cells cultured with or without bufalin and found miR148a to be the target of bufalin. miRNA expression profiling was performed in osteosarcomas by some researchers, but miR148a was not found to be significantly changed $[40,41]$. It suggests that miR148a specifically mediates the effect of bufalin on osteosarcoma cancer stem cells.

DNA methyltransferases (DNMTs) was reported to be upregulated in cisplatin-resistant ovarian cancer [42]. One of the most widely used DNA methyltransferase inhibitors, decitabine (5-aza-2'-deoxycytidine, DAC), was also reported to have effects in targeting the human osteosarcoma HosDXR150 cell line [43]. Here we also found that DNMT1 severed as the target of mi-148a. The separated and combined effects of bufalin and DAC on osteosarcoma cancer stem cells are worth exploring in a future study.

In conclusion, we established a new and efficient approach for the establishment of a patient-derived cancer cell line, and we isolated CSCs from the primary cell line. Moreover, we found that bufalin was a potent agent in inhibiting the proliferation and differentiation of C10S-derived CSCs. By examining the miRNA expression profile of C1OS cultured with or without bufalin, we identified miR-148a as a target that mediates the effects of bufalin. The downstream target of miR148a, DNMT1, was also identified in C1OS derived cancer stem cells. We further identified the function of bufalin, i.e., that it plays a role in the miR148a and DNMT1 pathway in osteosarcoma patients and targets p27 as a downstream signal of DNMT1. Through an in-depth study of biological characteristics and related signal pathways to identify specific targets, our data may offer hope for eradicating osteosarcoma using this compound.

\section{Acknowledgements}

This work is supported by grant from Natural Science Foundation of Shanghai (13ZR1442500).

\section{KARGER}




\section{Cellular Physiology Cell Physiol Biochem 2015;36:1186-1196 \begin{tabular}{l|l} 
DOI: 10.1159/000430289 & (C) 2015 S. Karger AG, Basel
\end{tabular} www.karger.com/cpb \\ Chang et al:: Bufalin Inhibits Cars from Os Cells Through Mir-148a}

\section{Disclosure Statement}

No potential conflict of interest was declared.

\section{References}

1 Picci P: Osteosarcoma (osteogenic sarcoma). Orphanet J Rare Dis 2007;2:6.

2 Ottaviani G, Jaffe N: The epidemiology of osteosarcoma. Cancer Treat Res 2009;152:3-13.

3 Ek ET, Dass CR, Choong PF: Commonly used mouse models of osteosarcoma. Crit Rev Oncol Hematol 2006;60:1-8.

4 Chou AJ, Gorlick R: Chemotherapy resistance in osteosarcoma: current challenges and future directions. Expert Rev Anticancer Ther 2006;6:1075-1085.

5 Longhi A, Errani C, De Paolis M, Mercuri M, Bacci G: Primary bone osteosarcoma in the pediatric age: state of the art. Cancer Treat Rev 2006;32:423-436.

6 Ta HT, Dass CR, Choong PF, Dunstan DE: Osteosarcoma treatment: state of the art. Cancer Metastasis Rev 2009;28:247-263.

7 Luo XJ, Tang DG, Gao TL, Zhang YL, Wang M, Quan ZX, Chen J: MicroRNA-212 inhibits osteosarcoma cells proliferation and invasion by down-regulation of Sox4. Cell Physiol Biochem 2014;34:2180-2188.

8 Xu H, Liu X, Zhao J: Down-regulation of miR-3928 promoted osteosarcoma growth. Cell Physiol Biochem 2014;33:1547-1556.

9 Xu G, Wang J, Jia Y, Shen F, Han W, Kang Y: MiR-142-3p functions as a potential tumor suppressor in human osteosarcoma by targeting HMGA1. Cell Physiol Biochem 2014;33:1329-1339.

10 Pan W, Wang H, Jianwei R, Ye Z: MicroRNA-27a promotes proliferation, migration and invasion by targeting MAP2K4 in human osteosarcoma cells. Cell Physiol Biochem 2014;33:402-412.

11 Li C, Heidt DG, Dalerba P, Burant CF, Zhang L, Adsay V, Wicha M, Clarke MF, Simeone DM: Identification of pancreatic cancer stem cells. Cancer Res 2007;67:1030-1037.

12 Costello RT, Mallet F, Gaugler B, Sainty D, Arnoulet C, Gastaut JA, Olive D: Human acute myeloid leukemia CD34+/CD38- progenitor cells have decreased sensitivity to chemotherapy and Fas-induced apoptosis, reduced immunogenicity, and impaired dendritic cell transformation capacities. Cancer Res 2000;60:44034411.

13 Dean M, Fojo T, Bates S: Tumour stem cells and drug resistance. Nat Rev Cancer 2005;5:275-284.

14 Di Fiore R, Santulli A, Ferrante RD, Giuliano M, De Blasio A, Messina C, Pirozzi G, Tirino V, Tesoriere G, Vento R: Identification and expansion of human osteosarcoma-cancer-stem cells by long-term 3-aminobenzamide treatment. J Cell Physiol 2009;219:301-313.

15 Wang L, Park P, Lin CY: Characterization of stem cell attributes in human osteosarcoma cell lines. Cancer Biol Ther 2009;8:543-552.

16 Zhang H, Wu H, Zheng J, Yu P, Xu L, Jiang P, Gao J, Wang H, Zhang Y: Transforming growth factor beta1 signal is crucial for dedifferentiation of cancer cells to cancer stem cells in osteosarcoma. Stem Cells 2013;31:433446.

17 Martins-Neves SR, Lopes AO, do Carmo A, Paiva AA, Simoes PC, Abrunhosa AJ, Gomes CM: Therapeutic implications of an enriched cancer stem-like cell population in a human osteosarcoma cell line. BMC Cancer 2012;12:139.

18 Chang Y, Zhao Y, Zhan H, Wei X, Liu T, Zheng B: Bufalin inhibits the differentiation and proliferation of human osteosarcoma cell line hMG63-derived cancer stem cells. Tumour Biol 2014;35:1075-1082.

19 Han KQ, Huang G, Gu W, Su YH, Huang XQ Ling CQ: Anti-tumor activities and apoptosis-regulated mechanisms of bufalin on the orthotopic transplantation tumor model of human hepatocellular carcinoma in nude mice. World J Gastroenterol 2007;13:3374-3379.

20 Watabe M, Ito K, Masuda Y, Nakajo S, Nakaya K: Activation of AP-1 is required for bufalin-induced apoptosis in human leukemia U937 cells. Oncogene 1998;16:779-787.

21 Yeh JY, Huang WJ, Kan SF, Wang PS: Effects of bufalin and cinobufagin on the proliferation of androgen dependent and independent prostate cancer cells. Prostate 2003;54:112-124.

22 Li D, Qu X, Hou K, Zhang Y, Dong Q, Teng Y, Zhang J, Liu Y: PI3K/Akt is involved in bufalin-induced apoptosis in gastric cancer cells. Anticancer Drugs 2009;20:59-64. 


\section{Cellular Physiology Cell Physiol Biochem 2015;36:1186-1196 \begin{tabular}{l|l} 
DOI: 10.1159/000430289 & (C) 2015 S. Karger AG, Basel
\end{tabular} and Biochemistry Published online: June 25, $2015 \quad$ www.karger.com/cpb \\ Chang et al.: Bufalin Inhibits Cars from Os Cells Through Mir-148a}

23 Wang J, Yin JQ Jia Q Shen JN, Huang G, Xie XB, Zou CY: [Bufalin induces apoptosis in osteosarcoma U-2OS and U-2OS methotrexate 300-resistant cell lines in vitro]. Zhonghua Zhong Liu Za Zhi 2010;32:734-738.

24 Jovanovic M, Hengartner MO: miRNAs and apoptosis: RNAs to die for. Oncogene 2006;25:6176-6187.

25 van Jaarsveld MT, Wouters MD, Boersma AW, Smid M, van Ijcken WF, Mathijssen RH, Hoeijmakers JH, Martens JW, van Laere S, Wiemer EA, Pothof J: DNA damage responsive microRNAs misexpressed in human cancer modulate therapy sensitivity. Mol Oncol 2014;8:458-468.

26 Xu M, Jin H, Xu CX, Bi WZ, Wang Y: MiR-34c inhibits osteosarcoma metastasis and chemoresistance. Med Oncol 2014;31:972.

27 Zhou Y, Huang Z, Wu S, Zang X, Liu M, Shi J: miR-33a is up-regulated in chemoresistant osteosarcoma and promotes osteosarcoma cell resistance to cisplatin by down-regulating TWIST. J Exp Clin Cancer Res 2014;33:12.

28 Ziyan W, Shuhua Y, Xiufang W, Xiaoyun L: MicroRNA-21 is involved in osteosarcoma cell invasion and migration. Med Oncol 2011;28:1469-1474.

29 Zhang H, Cai X, Wang Y, Tang H, Tong D, Ji F: microRNA-143, down-regulated in osteosarcoma, promotes apoptosis and suppresses tumorigenicity by targeting Bcl-2. Oncol Rep 2010;24:1363-1369.

30 Song B, Wang Y, Xi Y, Kudo K, Bruheim S, Botchkina GI, Gavin E, Wan Y, Formentini A, Kornmann M, Fodstad O, Ju J: Mechanism of chemoresistance mediated by miR-140 in human osteosarcoma and colon cancer cells. Oncogene 2009;28:4065-4074.

31 Li J, Zhong XY, Li ZY, Cai JF, Zou L, Li JM, Yang T, Liu W: CD133 expression in osteosarcoma and derivation of CD133(+) cells. Mol Med Rep 2013;7:577-584.

32 Di Fiore R, Fanale D, Drago-Ferrante R, Chiaradonna F, Giuliano M, De Blasio A, Amodeo V, Corsini LR, Bazan V, Tesoriere G, Vento R, Russo A: Genetic and molecular characterization of the human osteosarcoma 3ABOS cancer stem cell line: a possible model for studying osteosarcoma origin and stemness. J Cell Physiol 2013;228:1189-1201.

33 Mohseny AB, Machado I, Cai Y, Schaefer KL, Serra M, Hogendoorn PC, Llombart-Bosch A, Cleton-Jansen AM: Functional characterization of osteosarcoma cell lines provides representative models to study the human disease. Lab Invest 2011;91:1195-1205.

34 Gillet JP, Calcagno AM, Varma S, Marino M, Green LJ, Vora MI, Patel C, Orina JN, Eliseeva TA, Singal V, Padmanabhan R, Davidson B, Ganapathi R, Sood AK, Rueda BR, Ambudkar SV, Gottesman MM: Redefining the relevance of established cancer cell lines to the study of mechanisms of clinical anti-cancer drug resistance. Proc Natl Acad Sci U S A 2011;108:18708-18713.

35 Gazdar AF, Gao B, Minna JD: Lung cancer cell lines: Useless artifacts or invaluable tools for medical science? Lung Cancer 2010;68:309-318.

36 Schilsky RL: Personalized medicine in oncology: the future is now. Nat Rev Drug Discov 2010;9:363-366.

37 Mitsiades CS, Davies FE, Laubach JP, Joshua D, San Miguel J, Anderson KC, Richardson PG: Future directions of next-generation novel therapies, combination approaches, and the development of personalized medicine in myeloma. J Clin Oncol 2011;29:1916-1923.

38 Trusheim MR, Burgess B, Hu SX, Long T, Averbuch SD, Flynn AA, Lieftucht A, Mazumder A, Milloy J, Shaw PM, Swank D, Wang J, Berndt ER, Goodsaid F, Palmer MC: Quantifying factors for the success of stratified medicine. Nat Rev Drug Discov 2011;10:817-833.

39 Nana-Sinkam SP, Croce CM: MicroRNAs as therapeutic targets in cancer. Transl Res 2011;157:216-225.

40 Jones KB, Salah Z, Del Mare S, Galasso M, Gaudio E, Nuovo GJ, Lovat F, LeBlanc K, Palatini J, Randall RL, Volinia S, Stein GS, Croce CM, Lian JB, Aqeilan RI: miRNA signatures associate with pathogenesis and progression of osteosarcoma. Cancer Res 2012;72:1865-1877.

41 Maire G, Martin JW, Yoshimoto M, Chilton-MacNeill S, Zielenska M, Squire JA: Analysis of miRNA-gene expression-genomic profiles reveals complex mechanisms of microRNA deregulation in osteosarcoma. Cancer Genet 2011;204:138-146.

42 Li M, Balch C, Montgomery JS, Jeong M, Chung JH, Yan P, Huang TH, Kim S, Nephew KP: Integrated analysis of DNA methylation and gene expression reveals specific signaling pathways associated with platinum resistance in ovarian cancer. BMC Med Genomics 2009;2:34.

43 Capobianco E, Mora A, La Sala D, Roberti A, Zaki N, Badidi E, Taranta M, Cinti C: Separate and combined effects of DNMT and HDAC inhibitors in treating human multi-drug resistant osteosarcoma HosDXR150 cell line. PLoS One 2014;9:e95596. 